\title{
A EXPERIÊNCIA DA CONSTRUÇÃO DE UM PLANO OPERATIVO PARA O SETOR DE DEMANDA JUDICIAL NA SECRETARIA DE SAÚDE DO ESTADO DO PARANÁ
}

\section{CONSTRUCTION EXPERIENCE OF AN OPERATING PLAN FOR JUDICIAL DEMAND SECTOR OF HEALTH SECRETARY OF STATE OF PARANÁ}

\author{
IMAZU1, P.; OLIVEIRA', F. W.; CZEPULA², A.; STRAPASSON², G. C.
}

1 - Pós-graduandas da Universidade Federal de Santa Catarina. Curitiba - PR;

2 - Doutoras em Ciências Farmacêuticas pela Universidade Federal do Paraná. Curitiba - PR.

Autor para correspondência: pri_ima@yahoo.com.br

\section{RESUMO:}

Com o aumento da demanda judicial para fornecimento de medicamentos na Secretaria de Estado de Saúde do Paraná, a gestão do setor responsável tem se apresentado cada vez mais complexa. A ausência de legislação que regulamente parte da Assistência Farmacêutica (AF) dificulta mais a sua organização. Para enfrentamento dessas dificuldades foi elaborado um planejamento estratégico situacional (PES), conforme os fundamentos de Carlos Matus, seu idealizador. Este artigo relata uma experiência de elaboração de um plano operativo com quatro momentos, a saber: explicativo, normativo, estratégico e tático-operacional. No momento explicativo foi possível identificar os problemas com maior magnitude, transcendência, vulnerabilidade, urgência e factibilidade. Assim obteve-se por pontuação o problema a ser priorizado e estudado. As ações a serem realizadas para sua superação foram elencadas no momento normativo. No momento estratégico foi analisada a viabilidade e factibilidade de cada ação e atribuída atividade estratégica para aquelas que possuíam déficit. No momento tático-operacional foi elaborado o protocolo de avaliação, com indicadores e fontes de verificação para os resultados das ações. Por meio desse trabalho foi possível observar que o método de Carlos Matus é bem trabalhoso e exige bastante tempo para sua elaboração. Destacam-se como aspectos favoráveis: o foco a ser definido, o qual deve ser estudado antes do inicio do projeto, considerando o seu centralizador e o impacto que se almeja nas ações a serem realizadas; e a participação de atores internos e externos, que possibilitam o envolvimento direto de todos com o problema.

Palavras-chave: Planejamento Estratégico Situacional, Demandas Judiciais, SUS.

\section{ABSTRACT:}

With the increased demand for judicial supply of medicines in the State Department of Health of Paraná, responsible sector management has been performing increasingly complex. The absence of legislation regulating this part of pharmaceutical care (PC) further complicates your organization. To face these difficulties has been prepared a situational strategic planning (PSC) as the foundations of Carlos Matus, its creator. This article reports a development experience of an operating plan with four stages, namely: explanatory, normative, strategic and tactical-operational. In the explanatory moment it was possible to identify problems of greater magnitude, transcendence, vulnerability, urgency and feasibility. Thus was obtained by scoring the problem to be prioritized and studied. The actions to be 
taken to overcome them were listed in the normative time. In strategic moment it analyzed the viability and feasibility of each action and assigned strategic activity for those who had deficit. Then the tactical-operational time was prepared the evaluation protocol, with indicators and verifiers for the results of actions. Through this work it was observed that the Carlos Matus method is very labor intensive and requires considerable time for its preparation. Stand out as positive aspects: the focus to be set, which should be studied before the start of the project, considering its centralizer and the impact that crave the action to be taken; and the participation of internal and external actors that enable the direct involvement of all with the problem.

Keywords: Situational Strategic Planning, Legal Demands, SUS.

\section{INTRODUÇÃO}

O direito à saúde está previsto na Constituição Federal de 1988 (Artigo 6ํ) como direito fundamental garantido pelo estado. Além disso, a Lei ํo 8.080/90 regulamenta o Sistema Único de Saúde (SUS) estabelecendo como princípios a universalidade, igualdade e equidade do acesso aos serviços. O SUS prevê assistência terapêutica integral, inclusive farmacêutica (FIGUEIREDO; PEPE; OSORIO-DE-CASTRO, 2007).

A judicialização da saúde é, portanto, a "intervenção do Poder Judiciário na determinação do fornecimento, pelo Estado, de serviços e produtos destinados à saúde, independentemente das políticas públicas com esse fim" (ISABEL DOS SANTOS; SOARES, 2013).

Atualmente, a maioria das ações para saúde existentes é relacionada ao fornecimento de medicamentos. Os medicamentos mais demandados são aqueles não padronizados no SUS, de alto custo ou ainda os que não possuem registro no Brasil junto à Agência Nacional de Vigilância Sanitária (ANVISA) (WANG et al, 2014). Entretanto, não é incomum encontrar ações judiciais para fornecimento de medicamentos padronizados na Relação Nacional de Medicamentos Essenciais (RENAME) e pertencentes aos componentes básico, estratégico e especializado da Assistência Farmacêutica.

Esse fornecimento via judicial não está contemplado em nenhum componente da Assistência Farmacêutica (AF) no SUS, também não é regulamentado por nenhuma portaria ou legislação específica. Os medicamentos solicitados, os prazos para cumprimento das ordens judiciais e as condições de entrega podem variar muito. Além disso, as ações judiciais são muito específicas, o que dificulta a padronização de condutas, o planejamento e todo o ciclo da AF.

No estado do Paraná, com o crescimento das decisões judiciais para fornecimento de medicamentos, foi criado no Cemepar (Centro de Medicamentos do Paraná), no ano de 2005, o setor de Medicamentos por DJ (Demanda Judicial) através da SESA/PR (Secretaria da Saúde do Estado do Paraná). Medidas estruturais e organizacionais também foram criadas, como a definição de um fluxo interno, com o objetivo de enfrentar essas demandas 
de forma organizada, articulada e em parceria com a Assessoria Jurídica (AJU), Procuradoria Geral do Estado (PGE) e Regionais de Saúde do Estado (PONTAROLLI; SILVA; STRAPASSON, 2015).

Dos anos seguintes até os dias de hoje a demanda vem crescendo exponencialmente. Com isso, as atividades do setor se tornaram cada vez mais complexas e, associado a isso, o crescimento dos problemas e dificuldades. A DJ está exigindo mais investimentos em estrutura, sistemas de informação, recursos humanos, treinamentos, entre outros.

Diante de tal condição, um Plano Operativo para a Demanda Judicial na SESA/PR se faz necessário com certa urgência, para que a Assistência Farmacêutica nessa área também seja contemplada como foco para melhoria no atendimento do usuário, com consequente resultado na sua saúde.

Para isso, o Planejamento Estratégico Situacional (PES), desenvolvido por Carlos Matus, foi criado para ser utilizado na resolução de problemas complexos, como as organizações sociais, que possuem diversos tipos de incertezas e interferentes. Ele é baseado na estruturação e entendimento aprofundado de um problema complexo. O PES propõe a melhoria de um setor com a elaboração de diversas ações para enfrentamento das causas e consequências desses problemas, contando sempre que possível com a participação de diversos atores envolvidos (IIDA, 1993; NETO; JUNIOR; POLO, 2006).

No contexto da saúde, especialmente no Sistema Único de Saúde (SUS), o PES tem sido utilizado de diferentes maneiras. Conforme relatam Artmann, Azevedo e Castilho de Sá (1997), as organizações descentralizadas dos SUS possuem especificidades locais, que podem através desse enfoque metodológico serem compreendidas com maior profundidade.

Assim, o objetivo deste trabalho é relatar a experiência da construção de um plano operativo no setor de demanda judicial da SESA/PR, fazendo uma análise estruturada das etapas vivenciadas, das dificuldades e desafios, e das potencialidades do método aplicado.

\section{MÉTODOS}

Este artigo é um relato de experiência sobre a execução de um plano operativo PO com a utilização da metodologia de Carlos Matus.

As atividades do PO foram realizadas pela autora entre junho/2014 e fevereiro 2015, no setor de DJ, situado nas dependências do Cemepar. Também houve a participação de outros setores da SESA/PR, envolvidos direta e indiretamente com a DJ, como Assessoria Jurídica, Departamento de Assistência Farmacêutica, Almoxarifado, setor de compras, entre outros. 
O resultado se deu através da análise qualitativa das observações obtidas na construção dos quatro momentos do PO, a saber: explicativo, normativo, estratégico e tático-operacional. Também foram analisados artigos científicos relacionados ao assunto e disponíveis em diversas bases de dados gratuitas. Assim como Legislação Estadual e Sanitária atuais.

\section{RESULTADOS}

Para a realização do PES foi elaborado um plano operativo em diversas etapas.

Inicialmente, a equipe de farmacêuticos do setor decidiu pela realização de 3 PO com foco comum na demanda judicial da SESA/PR.

Para a definição dos atores foram analisados todos os envolvidos com 0 fornecimento de medicamentos por demanda judicial. Optou-se pela inclusão apenas daqueles que mantêm contato direto com o setor de DJ do Cemepar, tais como: advogados da Assessoria Jurídica da SESA/PR, Procuradores do Estado, Almoxarifado de Cemepar, Farmacêuticos das 22 Regionais de Saúde do Estado, Setor de Compras do Cemepar e Comissão Permanente de Licitação da SESA/PR.

A partir daí as etapas seguintes foram divididas em 4 momentos: explicativo, normativo, estratégico e tático-operacional.

\subsection{Momento Explicativo}

Para a elaboração do momento explicativo na DJ da SESA/PR foi realizada uma oficina para priorização dos problemas. Representantes de todos os atores acima mencionados foram convidados, entretanto participaram apenas os setores internos do Cemepar e Assessoria Jurídica da SESA. Também participaram representantes do Departamento de Assistência Farmacêutica (DEAF) e a direção do Cemepar.

Os problemas foram pontuados por cada participante quanto a sua magnitude, transcendência, vulnerabilidade, urgência e factibilidade. Segundo Barreto et al (2013), "Magnitude mede a frequência do problema e o número de pessoas por ele atingidas; transcendência mede o quanto as pessoas se importam com o problema; vulnerabilidade indica a facilidade de resolução do problema com os recursos disponíveis; urgência indica a percepção sobre qual o prazo existente para o enfrentamento do problema; e factibilidade mede os recursos disponíveis para a resolução do problema, incluindo recursos materiais, humanos, físicos, financeiros e políticos".

Os cálculos resultaram na priorização do problema "atraso no prazo para cumprimento das ordens judiciais". 
A explicação do problema priorizado se deu através da elaboração de uma espinha de peixe (FIGURA 1), um diagrama que facilita a visualização de causas e consequências de um determinado problema de uma organização. Nele foram elencados os descritores desse problema, ou seja, as evidências que o caracteriza. Com base nas suas diversas causas e consequências se estabeleceu o objetivo geral e imagem-objetivo do PO, a partir da convergência de informações.

Figura 1: Espinha de peixe

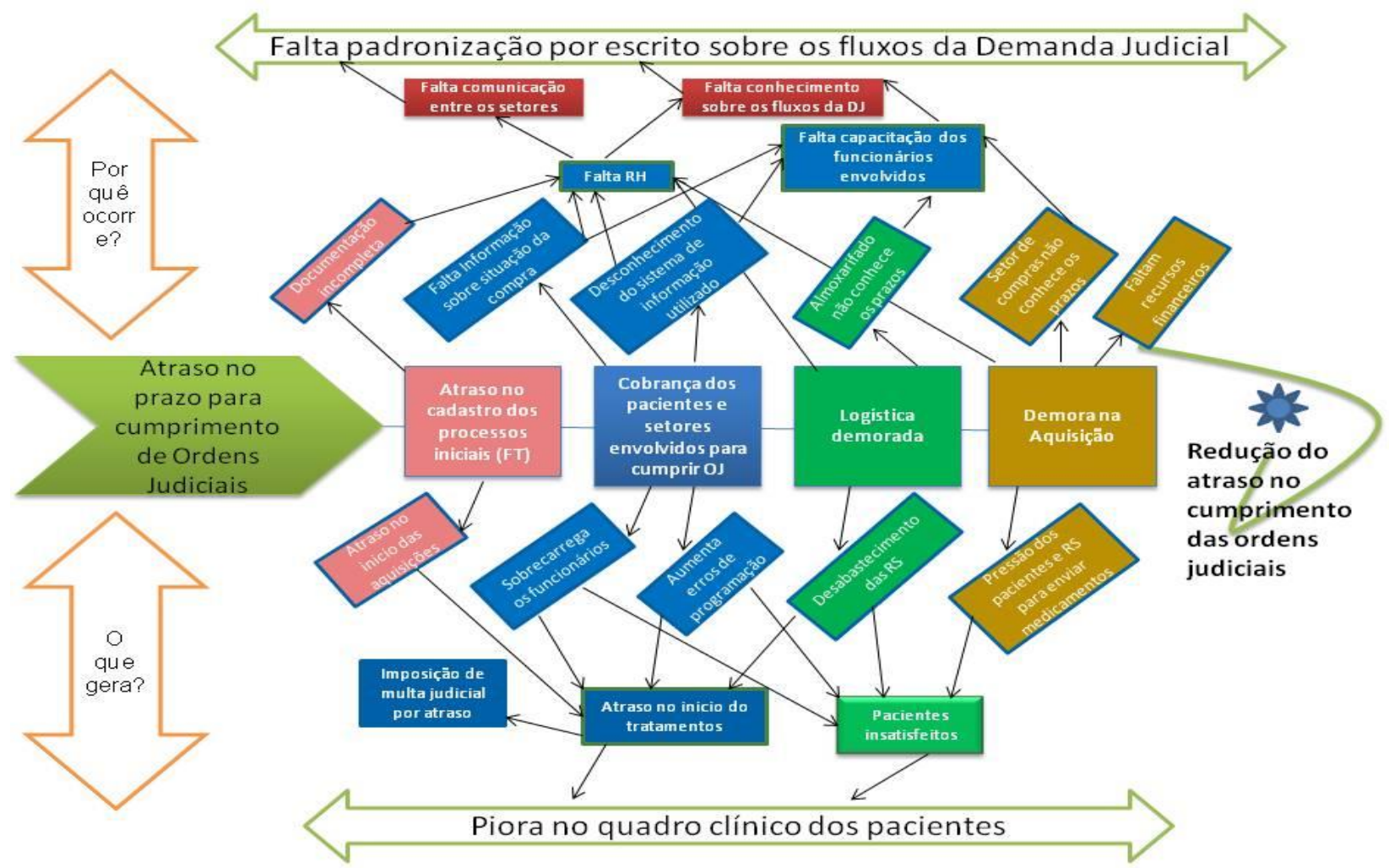

\subsection{Momento Normativo}

A partir da causa convergente "Falta padronização por escrito sobre os fluxos da Demanda Judicial" e da consequência convergente "Piora no quadro clínico dos pacientes", desenvolvidos durante o momento explicativo, foi definido o seguinte Objetivo Geral: "Padronizar por escrito os fluxos da demanda judicial, melhorando, assim, o quadro clínico dos pacientes".

O momento normativo é a elaboração do plano de intervenção, que irá solucionar o problema em questão (SANTANA et al ,2014). Esta etapa visa detalhar o objetivo geral através da delimitação de operações e ações com objetivos específicos. 
As operações e ações foram discutidas juntamente com os colegas do setor de DJ. De maneira geral, as atividades elencadas foram no sentido de melhorar a informação entre os envolvidos, tanto conferindo conhecimento sobre a atuação de cada autor, quanto melhorando o entendimento sobre o fluxo de da DJ, conforme QUADRO 1.

Quadro 1: resultados do momento normativo

\begin{tabular}{|c|c|c|}
\hline $\begin{array}{l}\text { OBJETIVOS } \\
\text { ESPECÍFICOS }\end{array}$ & OPERAÇÕES & AÇÕES \\
\hline \multirow{6}{*}{$\begin{array}{l}\text { Promover a } \\
\text { comunicação entre os } \\
\text { setores envolvidos. }\end{array}$} & \multirow{3}{*}{ Apresentação dos envolvidos. } & Definir as atribuições de cada setor. \\
\hline & & Relacionar os responsáveis/chefia de cada setor. \\
\hline & & $\begin{array}{l}\text { Listar contatos (telefone/e-mail) de todos os } \\
\text { envolvidos. }\end{array}$ \\
\hline & \multirow{3}{*}{$\begin{array}{l}\text { Criar Guia/Manual para uso do } \\
\text { sistema de informação utilizado. }\end{array}$} & $\begin{array}{l}\text { Levantar as principais dúvidas e problemas ocorridos } \\
\text { em relação ao sistema de informação. }\end{array}$ \\
\hline & & Escrever um guia/manual prático para uso do sistema. \\
\hline & & Realizar treinamento sobre o sistema. \\
\hline \multirow{7}{*}{$\begin{array}{c}\text { Promover a } \\
\text { capacitação dos } \\
\text { funcionários dos } \\
\text { diversos setores sobre } \\
\text { os fluxos da Demanda } \\
\text { Judicial. }\end{array}$} & \multirow{4}{*}{$\begin{array}{l}\text { Criar instruções de trabalho/fluxos } \\
\text { para as Regionais de Saúde (RS). }\end{array}$} & $\begin{array}{l}\text { Verificar os procedimentos escritos já existentes nas } \\
\text { RS. }\end{array}$ \\
\hline & & $\begin{array}{l}\text { Discutir com os envolvidos e chefias o conteúdo das } \\
\text { instruções e fluxo. }\end{array}$ \\
\hline & & Escrever as instruções de trabalho. \\
\hline & & Realizar treinamentos com os envolvidos. \\
\hline & \multirow{3}{*}{$\begin{array}{l}\text { Criar instruções de trabalho/fluxos } \\
\text { para o Cemepar. }\end{array}$} & $\begin{array}{l}\text { Revisar instruções de trabalho já existentes no } \\
\text { Cemepar. }\end{array}$ \\
\hline & & Elaborar novas instruções de trabalho. \\
\hline & & Realiza treinamento com os envolvidos. \\
\hline
\end{tabular}

\subsection{Momento Estratégico}

O momento estratégico visa identificar, nas ações estabelecidas no momento normativo, aquelas que não estão em condições de serem executadas, ou seja, os déficits das operações. Essa análise é feita através da avaliação dos recursos políticos, econômicos e administrativos (SANTANA et al.,2014; KLEBA; KRAUSER; VENDRUSCOLO, 2011).

Uma ação foi considerada viável quando o próprio setor de DJ apresenta poder para decidir, executar e manter. Foi factível quando os recursos necessários são existentes para aquele que pretende realizar a ação. Dessa maneira a matriz resultante apresentou 2 tipos de déficits em 5 ações diferentes (QUADRO 2). 
Quadro 2: resumo do momento estratégico

\begin{tabular}{|l|l|l|}
\hline \multicolumn{1}{|c|}{ AÇÕES } & \multicolumn{1}{|c|}{ DÉFICIT } & \multicolumn{1}{|c|}{ ATIVIDADE ESTRATÉGICA } \\
\hline $\begin{array}{l}\text { Definir as atribuições de cada } \\
\text { setor. }\end{array}$ & $\begin{array}{l}\text { Falta de recursos de } \\
\text { poder }\end{array}$ & $\begin{array}{l}\text { Sensibilizar aqueles que têm poder (chefias) } \\
\text { dentro dos diversos setores envolvidos com a } \\
\text { Demanda Judicial para definir e atuar na } \\
\text { operação. }\end{array}$ \\
\hline $\begin{array}{l}\text { Realizar treinamento sobre o } \\
\text { sistema. }\end{array}$ & $\begin{array}{l}\text { Falta de recursos } \\
\text { financeiros }\end{array}$ & $\begin{array}{l}\text { Sensibilizar a SESA/PR, os diretores das } \\
\text { Regionais de Saúde e do Cemepar quanto à } \\
\text { importância dos treinamentos para um bom } \\
\text { funcionamento da Demanda Judicial no estado. }\end{array}$ \\
\hline $\begin{array}{l}\text { Discutir com os envolvidos e conteúdo das } \\
\text { chefias o } \\
\text { instruções e fluxo. }\end{array}$ & $\begin{array}{l}\text { Falta de recursos de } \\
\text { poder }\end{array}$ & $\begin{array}{l}\text { Sensibilizar aqueles que têm poder (chefias) } \\
\text { dentro dos diversos setores envolvidos com a } \\
\text { Demanda Judicial para definir e atuar na } \\
\text { operação. }\end{array}$ \\
\hline $\begin{array}{l}\text { Realizar treinamentos com os } \\
\text { envolvidos. }\end{array}$ & $\begin{array}{l}\text { Falta de recursos } \\
\text { financeiros }\end{array}$ & $\begin{array}{l}\text { Sensibilizar a SESA/PR, os diretores das } \\
\text { Regionais de Saúde e do Cemepar quanto à } \\
\text { importância dos treinamentos para um bom } \\
\text { funcionamento da Demanda Judicial no estado. }\end{array}$ \\
\hline $\begin{array}{l}\text { Realvidos. } \\
\text { freinamento com os de recursos de } \\
\text { poder }\end{array}$ & $\begin{array}{l}\text { Sensibilizar a chefia do setor de DJ e demais } \\
\text { à importância dos treinamentos para um bom } \\
\text { resultado no cumprimento das ordens judiciais. }\end{array}$ \\
\hline
\end{tabular}

Resumindo, os dois tipos de déficits identificados foram: a falta de recursos de poder e falta de recursos financeiros. Para ambos, a atividade estratégica estabelecida foi a de sensibilizar àqueles que possuem poder, tanto para decidir, executar e manter, como para disponibilizar verba para a execução das ações.

\subsection{Momento Tático-operacional}

Para finalizar o plano operativo, foi necessário o estabelecimento de indicadores de monitoramento e avaliação para o acompanhamento de sua execução. Estes indicadores foram definidos por meio de um protocolo de indicadores.

A operação $n^{\circ} 1$ "Apresentação dos envolvidos", estabelecida no momento normativo apresentou para as suas ações um indicador e uma fonte de verificação concreto, conforme resume o Quadro 3 abaixo: 
Quadro 3: Momento tático-operacional (parte da tabela)

\begin{tabular}{|c|c|c|}
\hline Ação & Indicador & Fonte de verificação \\
\hline $\begin{array}{c}\text { Definir as atribuições de } \\
\text { cada setor. }\end{array}$ & $\begin{array}{c}\text { Ausência de conflitos de } \\
\text { atribuições. }\end{array}$ & $\begin{array}{c}\text { Atas de reuniões entre os setores } \\
\text { envolvidos. }\end{array}$ \\
\hline $\begin{array}{c}\text { Relacionar os } \\
\text { responsáveis/chefias de } \\
\text { cada setor. }\end{array}$ & $\begin{array}{c}\text { Lista contendo as } \\
\text { informações corretas e } \\
\text { atualizadas sobre os } \\
\text { responsáveis e chefia de } \\
\text { cada setor envolvido com a } \\
\text { Demanda Judicial. }\end{array}$ & $\begin{array}{c}\text { Planilhas da SESA com } \\
\text { farmacêuticos responsáveis nas } \\
\text { Regionais de Saúde e site da PGE } \\
\text { contendo os procuradores de cada } \\
\text { região do estado. }\end{array}$ \\
\hline $\begin{array}{c}\text { Listar contatos } \\
\text { (telefone/e-mail) de } \\
\text { todos os envolvidos. }\end{array}$ & $\begin{array}{c}\text { Lista contendo os contatos } \\
\text { corretos e atualizadas de } \\
\text { todos os envolvidos com a a } \\
\text { Demanda Judicial. }\end{array}$ & $\begin{array}{c}\text { Planilhas da SESA com todos os } \\
\text { contatos das Regionais de Saúde e } \\
\text { site da PGE contendo os contatos } \\
\text { dos procuradores de cada região do } \\
\text { estado. }\end{array}$ \\
\hline
\end{tabular}

Para as demais ações definidas no momento normativo, notou-se que os indicadores não possuem cálculo. As fontes de verificação são subjetivas, restringidas à análise qualitativa dos e-mails e telefonemas recebidos no setor, contendo solicitações de informação.

\section{DISCUSSÃO}

A escolha do foco do plano operativo é de extrema importância para o desenvolvimento de um PES com resultados alcançáveis. Um foco muito amplo, como o utilizado, e com participação de muitos setores externos ao foco, gerou durante a oficina de priorização a identificação de inúmeros problemas de difícil correção.

Conforme Kleba, Krause e Vendruscolo (2011), o momento explicativo é de fundamental importância para o desenvolvimento do PES, visto que é a etapa inicial onde é possível identificar, descrever e explicar os problemas de uma organização. É nele que ocorre o entendimento complexo da situação atual, as causas e as consequências dos descritores e como elas se relacionam. Com toda a informação obtida é possível então discutir as etapas seguintes de gestão para alcance da imagem-objetivo.

Dessa maneira, a realização da oficina por si só, deu indícios da real situação dos setores envolvidos. Durante as discussões, observou-se desconhecimento de algumas 
questões especificas em relação à DJ. Algumas dificuldades apontadas pelos participantes do próprio setor não eram reconhecidas pelos demais, e vice-versa. Essa troca de conhecimento entre os participantes nesse encontro facilitou a identificação mais clara dos problemas.

Entretanto, o "atraso no prazo para cumprimento das ordens judiciais" foi priorizado com grande aprovação dos atores. Isso, porque se trata de um problema que afeta diariamente todos os setores envolvidos.

Pepe et al (2010) relatam que um dos grandes problemas relacionados aos prazos judiciais está na concessão de tutela antecipada (liminar) pelo juízo, que implica na determinação da entrega imediata do medicamento. Isso ocorre sem a oitiva prévia do gestor, o que gera dificuldades na aquisição e entrega do produto ao paciente. Aliado à isso, a falta de planejamento também tem pesado, uma vez que não é possível prever o medicamento que será deferido nem sua quantidade. Assim, para compras emergenciais para atendimento de demanda judicial, muitas vezes o gestor acaba utilizando procedimentos de compra não usuais na administração pública, o que aumenta ainda mais os gastos, podendo ainda favorecer fraudes.

Esse "atraso" nos prazos processuais acarreta o adiamento do início do tratamento dos pacientes, que geralmente não possuem condições financeiras de arcar com os gastos em saúde. Associado a isso, ocorre imposição de multa judicial por descumprimento, o que também gera aumento de gastos públicos, atualmente escassos. Assim, o dia-a-dia dos setores fica sobrecarregado. A pressão sobre o setor de DJ na SESA/PR compromete e eficiência dos servidores com o volume crescente de ordens judiciais, o que também foi relatado pelos atores de outros setores.

Com relação à explicação do problema, observou-se que as causas dos descritores sempre envolvem o "desconhecimento" em uma parte do trabalho que cada setor realiza. Com isso, a causa convergente foi definida como a "falta de padronização por escrito sobre os fluxos da DJ".

Essa falta de padronização não é recente. Atualmente, existe apenas, em vigor, a Resolução Estadual n. 01 de 18 de junho de 2007, que padroniza os procedimentos relativos à aquisição de medicamentos para atender ordem judicial. Observa-se que ela cita de maneira geral que o fluxo se inicial com a elaboração do documento "ficha técnica" pela procuradoria geral do estado (PGE). Essa, encaminha o documento ao Cemepar, que irá iniciar processo de aquisição do medicamento. O Cemepar, por sua vez, deve enviar a medicação às Regionais de Saúde, que ficarão responsáveis pela dispensação ao paciente. 
Ou seja, essa resolução encontra-se desatualizada, pois fornece a base para a elaboração do fluxo do processo, entretanto não o detalha e deixa em aberto várias questões sobre a responsabilidade de cada ator.

Com isso, observou-se a necessidade de melhorar, através de padronização de procedimentos, a comunicação entre os setores e principalmente entre o poder judiciário e executivo. Segundo Pepe et al (2010), a questão até já foi levada para o Supremo Tribunal Federal, que, através da Resolução ํㅜㄴ 31/2010 do Conselho Nacional de Justiça, expressou objetivamente essa necessidade.

De fato, a DJ sempre foi carente de procedimentos por escrito e a sua elaboração juntamente com as ações delimitadas no momento normativo contribuirão certamente para a redução do atraso no cumprimento das ordens judiciais e desta forma será possível acelerar o acesso dos pacientes aos medicamentos requeridos. Já a consequência convergente não poderia ser nada além da "piora no quadro clínico dos pacientes". É importante ressaltar isso, visto que, mesmo que o fornecimento judicial de medicamentos não esteja regulamentado por nenhuma portaria, ou que muitas vezes seja moralmente questionável, ainda assim se trata de assistência farmacêutica, onde o foco deve estar na saúde do paciente e não somente nas questões burocráticas de cada processo.

Por se tratar de um PES realizado com fins didáticos e por ser o primeiro realizado no setor, as ações corretivas foram planejadas para serem executadas pelos próprios funcionários da DJ. Portanto, a partir do momento explicativo, não foi solicitado a participação dos atores externos. Nesse caso, as atividades a serem executadas foram restritas ao setor interno, não envolvendo atores como AJU e PGE.

Apesar das atividades executadas pela AJU e PGE serem muito semelhantes em relação ao atendimento das ações judiciais, a ausência da PGE na oficina de priorização pode ter limitado o entendimento de alguns problemas. Não podemos saber exatamente se isso chegaria a afetar a elaboração do PES.

No caso em questão, o problema "atraso no prazo para cumprimento das ordens judiciais" gerou através do PES ações corretivas muito restritas, que podem auxiliar na obtenção da imagem-objetivo, entretanto não trazem mudanças de alto impacto. Os déficits gerados foram subjetivos, não há, de forma explícita, a maneira de contorná-los, o que gera dúvidas sobre a execução das ações.

Portanto, conforme citado, um foco muito amplo, como o utilizado, seria mais adequado para os gestores possuidores de recursos de poder ou com grande influência sobre os mesmos. Já para um funcionário regular, não ocupante de cargo algum, o 
estabelecimento de um foco mais restrito, geraria mais possibilidades de ações com objetivos de impacto imediato e fácil manejo, o que muitas vezes é mais necessário do que as grandes mudanças.

Quer dizer, em muitos casos as mudanças mais drásticas de um setor só ocorreriam com um grande interesse dos gestores hierarquicamente superiores ao elaborador do PES. O déficit de recursos de poder é limitante para um funcionário comum, alheio às grandes chefias.

Além dos déficits, é importante destacar que toda a formalidade exigida pelos preceitos de Matus para o desenvolvimento de um PES é bem trabalhosa e despende muito tempo dos atores envolvidos.

$\mathrm{Na}$ experiência da DJ houve a realização de um grande evento para discussão e priorização dos problemas, mas o mesmo padrão não foi seguido para os demais momentos. Optou-se por realizar reuniões rápidas e mais objetivas do que uma oficina para o desenvolvimento dos momentos explicativo, normativo, estratégico e tático-operacional.

Artmann, Azevedo e Castilho de Sá (1997), já relatavam a importância da velocidade na capacidade de respostas aos problemas no SUS. Os autores descrevem que é necessário evitar o descompasso entre o tempo necessário para sistematização e necessidade da resolução na dinâmica da organização. A otimização do tempo no PES para a DJ, se deu em parte pela falta de interesse dos atores externos à DJ e em parte pelas dificuldades logísticas de reunir todos num mesmo local. Ao contrário do que se esperava isso gerou frutos positivos na elaboração do PES, visto que ao longo de seu desenvolvimento pôde-se alterar os dados conforme os novos conhecimentos iam surgindo. Ou seja, deixou o PES mais maleável, dando liberdade ao gestor para fazer as adaptações necessárias conforme sua realidade.

Ainda assim, apesar das dificuldades encontradas no desenvolvimento do PES, o método se mostrou excelente para gestão da assistência farmacêutica. Os erros cometidos na sua elaboração e as dificuldades apresentadas foram de grande valia para 0 aprendizado e avaliação do método de Matus. Entretanto observa-se a necessidade de realizar outros PES com maior atuação dos autores externos ao Cemepar ou com focos mais específicos, para que o envolvimento seja maior e elaborem-se ações mais impactantes para o SUS. 


\section{REFERÊNCIAS}

ARTMANN, E.; AZEVEDO, C. da S.; SÁ, M. de C., Possibilidades de aplicação do enfoque estratégico de planejamento no nível local de saúde: análise comparada de duas experiências. Cad. Saúde Públ., Rio de Janeiro, v. 13, n. 4, p. 723-740, 1997.

BARRETO, J. L. et al. Operacionalização de um processo de planejamento. In: Una-SUS: Gestão da Assistência Farmacêutica - Ead. Florianópolis, 2013

CAVALCANTE, B. L. de L.; LIMA, U. T. S., Relato de experiência de uma estudante de enfermagem em um consultório especializado em tratamento de feridas. Journal of Nursing and Health, Pelotas, v. 1, n. 2, p. 94-103, 2012.

FIGUEIREDO, T. A.; PEPE, V. L. E.; OSORIO-DE-CASTRO, C. G. S., Um enfoque sanitário sobre a demanda judicial de medicamentos. Physis Revista de Saúde Coletiva, Rio de Janeiro, v. 20, n 1, p. 101-118, 2010.

HERMANN, A. P.; LACERDA, M. R., Atendimento domiciliar à saúde: um relato de experiência. Cogitare Enferm, Curitiba, v. 12, n. 4, 2007.

IIDA, I., Planejamento Estratégico Situacional. Produção, São Paulo, v. 3, n. 2, 1993.

ISABEL DOS SANTOS, R.; SOARES, L. Saúde e Cidadania. In: Una-SUS: Gestão da Assistência Farmacêutica - Ead. Florianópolis, 2013.

KLEBA, M. E.; KRAUSER, I. M.; VENDRUSCOLO, C., O planejamento estratégico situacional no ensino da gestão em saúde da família, Texto \& Contexto, Florianópolis, v. 20, n. 1, 2011.

NETO, F. S; JUNIOR, F. H; POLO, E. F., A adoção do modelo de planejamento estratégico situacional no setor público brasileiro: um estudo de caso. Organizações \& Sociedade, Salvador, v. 13, n. 39, 2006.

PARANÁ. Resolução n. 01 de 18 de junho de 2007. Padroniza os procedimentos relativos 
à aquisição de medicamentos para atender ordem judicial. Diário Oficial do Estado do Paraná, Curitiba, PR, 18 de jun. 2007. p. 11.

PEPE, V. L. E. et al. A judicialização da saúde e os novos desafios da gestão da assistência farmacêutica. Ciência \& Saúde Coletiva, Rio de Janeiro, v. 15, n. 5, p. 2405-2414, 2010.

PONTAROLLI, D. R. S.; SILVA, G. R. P. da P.; STRAPASSON, G. C.; O enfrentamento das demandas judiciais por medicamentos na secretaria de estado da saúde do Paraná. Direito à Saúde. Conselho Nacional de Secretários da Saúde, Brasília, 2015.

RIVEIRA, F. J. U., A gestão situacional (em saúde) e a organização comunicante. Cad. Saúde Públ., Rio de Janeiro, v. 12, n. 3, p. 357-352, 1996.

SANTANA, R. S. et al., A institucionalização da seleção de medicamentos em hospitais públicos por meio do planejamento estratégico situacional. Rev. Adm. Pública, Rio de Janeiro, v. 48, n. 6, p. 1587-1603, 2014.

WANG, D. W. et al. Os impactos da judicialização da saúde no município de São Paulo: gasto público e organização federativa. Rev. Adm. Pública, Rio de Janeiro, v. 48, n. 5, p. 1191-1206, 2014. 